\title{
Risk markers of all-cause and diagnosis-specific disability pension - a prospective cohort study of individuals sickness absent due to stress-related mental disorders
}

Kazi Ishtiak-Ahmed ${ }^{1,2}$, Aleksander Perski ${ }^{3}$ and Ellenor Mittendorfer-Rutz ${ }^{1 *}$

\begin{abstract}
Background: Stress-related mental disorders rank among the leading causes of sickness absence in several European countries. The aim of this study was to investigate predictors of all-cause and diagnosis-specific disability pension in sickness absentees with stress-related mental disorders.

Methods: A cohort of 36304 non-retired individuals aged 16-64 years at 31.12.2004 with at-least one sickness absence spell due to stress-related mental disorders (SRMD) initiated in 2005 in Sweden was followed-up with regard to disability pension (2006-2010) by linkage of registers. Uni- and multivariate Hazard ratios (HR) with 95\% Confidence Intervals, $\mathrm{Cl}$, were estimated using Cox regression for several risk markers.

Results: During the follow-up period, 2735 individuals (7.5\%) were granted a disability pension, predominantly due to mental diagnoses $(n=2004,73.3 \%)$. In the multivariate analyses, female sex, age exceeding 35 years, low educational level, being born in a country outside EU25 and Northern Europe, residing outside big cities, living alone, having had a long duration of the first spell due to SRMD ( $>90$ days); mental disorders necessitating frequent specialised health care as well as comorbid somatic disorders were found to be predictive of granting disability pension. Some different patterns emerged for risk factors related to diagnosis-specific disability pension and for younger and older individuals.
\end{abstract}

Conclusions: Several predictors could be identified as risk markers for disability pension. The variation in the effect of risk markers with regard to age and diagnosis of disability pension speaks in favour of the importance of a person-centered approach in treatment and rehabilitation.

Keywords: Stress-related mental disorders, Disability pension, Somatic disorders, Sickness absence

\section{Background}

Sickness absence due to mental disorders and particularly due to stress-related mental disorders represents a major public health problem in many European countries [1]. Over the last two decades, the proportion of individuals sickness absent due to mental disorders among all sickness absentees has been increasing in most of European countries [1]. In Sweden, stress-related mental disorder (SRMDs) was one of the single most frequent

\footnotetext{
* Correspondence: ellenor.mittendorfer-rutz@ki.se

'Division of Insurance Medicine, Department of Clinical Neuroscience, Karolinska Institutet, Berzelius väg 3, SE- 17177 Stockholm, Sweden Full list of author information is available at the end of the article
}

sickness absence diagnoses in initiated sickness absence spells in 2012 [2]. Stress-related mental disorders correspond to the diagnostic code F43 in the International Classification of Diseases (ICD, version 10), and include reactions to severe stress and adjustment disorders [3].

Evidence suggests that the risk of granted disability pension (DP) is increased in patients sickness absent due to mental diagnoses [4-12]. Few of the existing studies, however, focused on investigating specific mental disorders $[7,13]$. Disability pension as part of the social insurance system is intended to cover economical shortages due to disease and injury [14]. Still, disability pension 
has also been associated with adverse health behavior and social isolation $[15,16]$. In order to reduce the number of individuals with mental disorders with early exit from the labour market, a recent OECD report highlights the importance of research regarding the identification of risk factors for disability pension in this group [17]. Despite the size of the public health problem of sickness absence due to stress-related mental disorders, we are not aware of any studies investigating predictors of diagnosis-specific disability pension in this group.

The study aimed to investigate the association of risk markers including socio-demographics factors, sickness absence and health care patterns to subsequent granted all-cause and diagnosis-specific DP in individuals sickness absent due to stress-related mental disorder.

\section{Methods}

This is a prospective cohort study effected through linking of Swedish registers. The study base comprised all individuals 16 to 64 years of age at 31.12.2004 and registered in Sweden with at least one sickness absence spell due to a stress-related mental disorder (SRMD) initiated in $2005(\mathrm{~N}=38872)$. In total, 2059 and 265 individuals were excluded as they were on disability or old-age pension before baseline (2005), respectively. Two individuals were excluded as they were erroneously coded as dead before 2005. In addition, individuals with missing information on educational status $(\mathrm{N}=41)$, and on the length of the first sickness absence spell due to SRMD ( $N=201)$ were excluded. Finally, 36304 individuals (26315 women and 9,989 men) were included in the study.

Data from the following registers were used for all individuals in the study: 1) Statistics Sweden: Longitudinal integration database for health insurance and labour market studies (LISA) with socio-demographic information on age, sex, country of birth, place of residence, family situation and educational status. 2) The National Board of Health and Welfare: (i) National patient register providing information on date and diagnosis of inand outpatient care, (ii) Cause of death register including data on date and cause of death. 3) The Social Insurance Agency (SIA): Micro-data for analyses of social insurance (MiDAS) with information on date, grade and diagnosis of sickness absence and disability pension. Linkages were based on the de-identified personal numbers of all residents in Sweden.

\section{Ethical considerations}

This study was approved by the regional ethical review board, Karolinska Institutet, Stockholm (Dnr 2007/762-31).

\section{The Swedish social insurance system}

In Sweden, all people above the age of 16, who live in the country and have an income from work or unemployment benefits, can receive sickness benefits if their work capacity is reduced due to disease or injury [18]. The employer pays sick pay for the first 14 days of a sick-leave spell, following a first qualifying day (without economical compensation). Thereafter, the Social Insurance Agency pays sickness benefit. Self-employed and unemployed people get all sickness benefits from the Social Insurance Agency. After seven days of self-certification, a physician certificate is required. In this study, Social Insurance Agency provided data on sick leave with benefits and data on disability pension. Individuals who have a medically certified illness or injury which contribute to a permanently reduced work capacity to at least $25 \%$ are eligible for being granted a disability pension before reaching the age for old-age retirement [19].

\section{Diagnostic criteria}

All diagnoses related to risk factors and outcomes were based on the corresponding codes of the International Classification of Diseases (ICD) in ICD 10. Mental diagnoses were defined as F00-F99 in ICD 10. Somatic diagnoses comprised all remaining diagnoses. The definition of stress-related mental disorders was based on the corresponding ICD 10 code F43.

\section{Measures of risk markers}

Baseline socio-demographic characteristics derived from the LISA database and measured at the end of 2004 were categorized as shown in Table 1 . Both variables on sickness absence relate to spells initiated in 2005. The variable on diagnosis-specific sickness absence spells was categorized as only spells due to mental disorders; one additional spell due to a somatic disorder and multiple additional spells due to somatic disorders. Also, a variable on the numbers of sick-leave spells due to mental diagnoses $(1,2$, $3+$ ) was categorised. Duration of the first sickness absence spell due to SRMD initiated in 2005 was grouped in five categories: $1-14,15-90,91-180,181-365$ and more than 365 days. Categorization of health care variables was based on the diagnosis-specific median number of days and number of visits in inpatient (2000-2005) and outpatient care (2001-2005), respectively (four variables). The median length of hospital stay due to mental and somatic diagnoses was eight and four days, respectively, while the median number of visits in outpatient care due to mental and somatic diagnoses reached one and four visits, respectively.

\section{Outcome measure}

Disability pension, was defined as granted all-cause and diagnosis-specific (mental and somatic) disability pension occurring during the follow-up period. 
Table 1 Descriptive statistics for individuals $(\mathrm{N}=36304)$ with sickness absence due to stress-related mental disorders (SRMD) initiated in 2005 and subsequent disability pension (DP, 2006-2010)

\begin{tabular}{|c|c|c|c|c|c|}
\hline \multirow[b]{2}{*}{ Characteristics } & \multicolumn{2}{|c|}{ All } & \multirow{2}{*}{$\begin{array}{r}\mathrm{DP} \\
\mathrm{N}, \% \\
\end{array}$} & \multirow{2}{*}{$\frac{\mathrm{DP}, \text { mental }}{\mathrm{N}, \%}$} & \multirow{2}{*}{$\frac{\mathrm{DP} \text {, somati }}{\mathrm{N}, \%}$} \\
\hline & $\mathbf{N}$ & $\%$ & & & \\
\hline \multicolumn{6}{|l|}{ Socio-demographic characteristics } \\
\hline \multicolumn{6}{|l|}{ Sex } \\
\hline Male & 9989 & 27.5 & $705(7.1)$ & $543(27.1)$ & $162(22.2)$ \\
\hline Female & 26315 & 72.5 & $2030(7.7)$ & $1461(72.9)$ & $569(77.8)$ \\
\hline \multicolumn{6}{|l|}{ Age (years) } \\
\hline $17-25$ & 1821 & 5.0 & $71(3.9)$ & $61(3.0)$ & $10(1.4)$ \\
\hline $26-35$ & 8216 & 22.6 & $313(3.8)$ & $261(13.0)$ & $52(7.1)$ \\
\hline $36-45$ & 11464 & 31.6 & $611(5.3)$ & $505(25.2)$ & $106(14.5)$ \\
\hline $46-55$ & 9138 & 25.2 & $794(8.7)$ & $564(28.1)$ & $230(31.5)$ \\
\hline $56-65$ & 5665 & 15.6 & $946(16.7)$ & $613(30.6)$ & $333(45.6)$ \\
\hline \multicolumn{6}{|l|}{ Education (years) } \\
\hline 0 to 9 & 4362 & 12.0 & $474(10.9)$ & $314(15.7)$ & $160(21.9)$ \\
\hline 10 to 12 & 18066 & 49.8 & $1292(7.2)$ & $921(46.0)$ & $371(50.8)$ \\
\hline Above 12 & 13876 & 38.2 & $969(7.0)$ & $769(38.4)$ & $200(27.4)$ \\
\hline \multicolumn{6}{|l|}{ Country of birth* } \\
\hline Sweden & 31763 & 87.5 & $2264(7.1)$ & $1637(81.7)$ & $627(85.8)$ \\
\hline Other Nordic countries & 1313 & 3.6 & $121(9.2)$ & $75(3.7)$ & $46(6.3)$ \\
\hline EU25 without Nordic countries & 717 & 2.0 & $57(7.9)$ & $51(2.5)$ & $6(0.8)$ \\
\hline Rest of the world & 2511 & 6.9 & $293(11.7)$ & $241(12.0)$ & $52(7.1)$ \\
\hline \multicolumn{6}{|l|}{ Place of residence ${ }^{* *}$} \\
\hline Big cities & 14017 & 38.6 & $911(6.5)$ & $734(36.6)$ & $177(24.2)$ \\
\hline Medium sized cities & 12869 & 35.4 & $1011(7.9)$ & $699(34.9)$ & $312(42.7)$ \\
\hline Small towns/villages & 9418 & 25.9 & $813(8.6)$ & $571(28.5)$ & $242(33.1)$ \\
\hline \multicolumn{6}{|l|}{ Type of family ${ }^{a}$} \\
\hline Married/cohabiting without children & 4663 & 12.8 & 619 (13.3) & $420(21.0)$ & $199(27.2)$ \\
\hline Married/cohabiting with children & 13955 & 38.4 & $795(5.7)$ & $600(29.9)$ & $195(26.7)$ \\
\hline Single people without children & 11490 & 31.6 & $911(7.9)$ & $671(33.5)$ & $240(32.8)$ \\
\hline Single people with children & 6196 & 17.1 & $410(6.6)$ & $313(15.6)$ & $97(13.3)$ \\
\hline \multicolumn{6}{|l|}{ Sickness absence characteristics ${ }^{b}$} \\
\hline \multicolumn{6}{|l|}{ Number of spells } \\
\hline Only mental & 32586 & 89.8 & $2310(7.1)$ & $1770(88.3)$ & $540(73.9)$ \\
\hline 1 spell somatic & 3330 & 9.2 & $377(11.3)$ & $210(10.5)$ & $167(22.8)$ \\
\hline$>1$ spell somatic & 388 & 1.1 & $48(12.4)$ & $24(1.2)$ & $24(3.3)$ \\
\hline \multicolumn{6}{|l|}{ Duration $\left(\right.$ days) ${ }^{c}$} \\
\hline $1-14$ & 10157 & 28.0 & $307(25)$ & $167(8.3)$ & $140(19.2)$ \\
\hline $15-90$ & 17370 & 47.8 & $575(3.3)$ & $338(16.9)$ & $237(32.4)$ \\
\hline $91-180$ & 3742 & 10.3 & $220(5.9)$ & $171(8.5)$ & $49(6.7)$ \\
\hline 181-365 & 2469 & 6.8 & $420(17.0)$ & $313(15.6)$ & $107(14.6)$ \\
\hline$>365$ & 2566 & 7.1 & $1213(47.3)$ & $1015(50.6)$ & $198(27.1)$ \\
\hline
\end{tabular}


Table 1 Descriptive statistics for individuals $(\mathrm{N}=36304)$ with sickness absence due to stress-related mental disorders (SRMD) initiated in 2005 and subsequent disability pension (DP, 2006-2010) (Continued)

\begin{tabular}{|c|c|c|c|c|c|}
\hline \multicolumn{6}{|c|}{ Length of hospital stay due to mental diagnoses (days) ${ }^{d}$} \\
\hline No hospital stay & 35036 & 96.5 & $2492(7.1)$ & $1782(88.9)$ & $710(97.1)$ \\
\hline 1 to 8 & 663 & 1.8 & $102(15.4)$ & $91(4.5)$ & $11(1.5)$ \\
\hline$>8$ & 605 & 1.7 & $141(23.3)$ & $131(6.5)$ & $10(1.4)$ \\
\hline \multicolumn{6}{|c|}{ Length of hospital stay due to somatic diagnoses (days) ${ }^{d}$} \\
\hline No hospital stay & 25619 & 70.6 & $1752(6.8)$ & $1334(66.6)$ & $418(57.2)$ \\
\hline 1 to 4 & 5797 & 16.0 & $528(9.1)$ & $374(18.7)$ & $154(21.1)$ \\
\hline$>4$ & 4888 & 13.5 & $455(9.3)$ & $296(14.8)$ & $159(21.8)$ \\
\hline \multicolumn{6}{|c|}{ Outpatient care visit due to mental diagnoses ${ }^{d}$} \\
\hline No visits & 33013 & 90.9 & $2242(6.8)$ & $1581(78.9)$ & $661(90.4)$ \\
\hline 1 & 1763 & 4.9 & $200(11.3)$ & $162(8.1)$ & $38(5.2)$ \\
\hline$>1$ & 1528 & 4.2 & $293(19.2)$ & $261(13.0)$ & $32(4.4)$ \\
\hline \multicolumn{6}{|c|}{ Outpatient care visit due to somatic diagnoses ${ }^{d}$} \\
\hline No visits & 8806 & 24.3 & $462(5.2)$ & $356(17.8)$ & $106(14.5)$ \\
\hline 1 to 4 & 16238 & 44.7 & $1075(6.6)$ & $795(39.7)$ & $280(38.3)$ \\
\hline$>4$ & 11260 & 31.0 & $1198(10.6)$ & $853(42.6)$ & $345(47.2)$ \\
\hline
\end{tabular}

\section{Statistical analysis}

Cox proportional hazard regression models were applied after assuring that the proportional hazard assumption was met. Individuals were followed from 01.01.2006 to the date of granted disability pension, death, emigration, 65 years of age or December $31^{\text {st }} 2010$, whichever came first. Univariate and multivariate Hazard ratios and 95\% Confidence intervals (CIs) were estimated with regard to disability pension for the various risk makers. In multivariate models all variables were mutually adjusted for each other. The partical likelihood ratio test was used to test for interaction with sex and age. Due to collinearity of the variable "number of mental sick-leave spells" with other sick-leave related covariates, a separate multivariate analysis was carried out including this variable adjusting for socio-demographics and health care determinants. All statistical analyses were conducted using SPSS software version 22 .

\section{Results}

Of the 36,304 individuals, nearly 3 times as many women (72.5\%) than men $(27.5 \%)$ were on sickness absence due to SRMD during 2005. The mean age of the study population was 42.9 years (standard deviation, SD: 10.7). The mean follow-up time was 4.6 years (SD:1.1). During follow-up, 317 (0.9) individuals died, while 249 (0.7) emigrated and 2380 (6.6) reached retirement age (65 years).

Descriptive statistic of individuals with a new sickleave spell due to SRMD in 2005 and subsequent granting of disability pension is presented in Table 1 . The majority of the study population had a medium level of education (50\%), was born in Sweden (88\%), lived in bigger cities (39\%), was married/cohabiting with children living at home (38\%), had only spells with mental diagnoses (90\%) and a duration of the first spell of 15 to 90 days of sickness absence (48\%). Most of them did not have a history of inpatient or outpatient care due to mental diagnoses (97 and 91\%, respectively) or inpatient care due to somatic diagnoses (71\%), but had between 1 and 4 outpatient care visits due to somatic diagnoses between 2001 and 2005 (45\%).

During the five years of follow-up, 2735 individuals (7.5\%) initially on sickness absence due to stress-related mental disorders were granted a disability pension (DP), the majority $(n=2004)$ due to mental and 731 due to somatic diagnoses. The main diagnoses for disability pension due to mental diagnoses were stress-related mental disorder (ICD 10 code, F43, $\mathrm{N}=937,47 \%$ ) and depressive episode (ICD 10 code F32, $\mathrm{N}=410,20 \%$ ), data not shown. The main diagnoses underlying the disability pension due to somatic diagnoses were 
musculoskeletal diagnoses (ICD 10 codes M00-99, $\mathrm{N}=284,39 \%$ ), data not shown.

The proportion of individuals who were granted a disability pension during follow-up was highest among those older than 55 years (17\%), with the lowest educational level (11\%), those born outside of EU25 or Northern Europe (12\%), living in small towns or villages (9\%), those living alone (8\%), and having multiple additional spells with somatic diagnoses (12\%), individuals who had sickleave spell duration exceeding one year (47\%), those with more than median days/visits in in- or outpatient care due to mental (23\% and 19\%) and somatic diagnoses (9\% and $11 \%)$, respectively.

\section{All-cause disability pension}

Univariate and mutually adjusted HRs and 95\% CIs for the various risk factors with regard to subsequent granting of disability pension are presented in Table 2 . With regard to the socio-demographic characteristics, female sex, being above 35 years of age, with a low level of education (up to 9 years of schooling), born in other Nordic countries (than Sweden) or outside of Europe (EU25/Nordic countries), living outside big cities or in a family situation other than married/cohabiting with children were associated with significantly higher risks of disability pension in the univariate analyses. The risk of granted disability pension was further increased in case of one or more additional sickness absence spells due to somatic diagnoses and if the duration of the first sickness absence spell due to SRMD exceeded 3 months. The HRs and 95\% CIs for one and more additional spells due to mental disorders were 1.36 (1.19-1.55) and 1.89 (1.28-2.78), respectively. The risk was also elevated with increasing number of days in in- and outpatient care due to mental or somatic diagnoses.

In the multivariate analysis, mutually adjusting for all risk markers, female sex, low educational level, country of birth outside Europe (EU25/Nordic countries), living outside big cities as well as living without children remained to be significant predictors. The risk of disability pension increased with increasing age at baseline. Individuals of more than 55 years of age had a much higher HR (5.30, 95\% CI 4.15-6.83) compared to those below 25 years of age. The risk increased with rising number of spells due to somatic diagnoses and with increasing duration of the first spell in the multivariate analysis. Also the risk associated with one additional mental sick-leave spell remained higher after multivariate adjustment, HR 1.17, CI 1.02-1.33. Inpatient care due to mental diagnoses and outpatient care due to mental and somatic diagnoses were signficantly associated with an increased risk of subsequent disability pension, regardless of length of hospital stay or number of visits.

\section{Disability pension due to mental and somatic diagnoses}

Table 3 shows multivariate adjusted Hazard ratios and 95\% Confidence Intervals for the various risk markers in relation to granted DP due to mental and somatic diagnoses, separately. Female sex and low educational level turned out to be significant predictors for DP due to somatic diagnoses, while living alone was predictive for DP due to mental diagnoses. The risk of granted DP increased with increasing age with higher risk estimates related to DP due to somatic diagnoses than due to mental diagnoses. For example, the HRs related to the age group 56 to 65 years (reference group comprised those between 17 and 25 years of age) for DP due to mental diagnoses and due to somatic diagnoses were $3.76,95 \%$ CI 2.86-4.95 and 14.59, 95\% CI 7.70-27.63, respectively (Table 3). Being born outside Europe (Northern Europe/EU25) turned out to be a similarly important risk marker for DP due to mental and somatic diagnoses, while being born in other Nordic countries than Sweden was only predictive of DP due to somatic diagnoses. Residing in small towns or villages as compared to big cities was associated with higher risk estimates for DP due to somatic than mental diagnoses.

With regard to the sickness absence characteristics, increasing duration of the first sickness absence spell due to SRMD was both strongly related to the risk of DP due to mental and somatic diagnoses, considerably stronger to DP due to mental diagnoses, though. Having had more than half a year of sickness absence was associated with a HR 7.89, 95\% CI 6.54-9.53 and HR 3.53, 95\% CI 2.74-4.54 for DP due to mental and somatic diagnoses compared to having absences up to 2 weeks, respectively. Also, having at least one spell due to somatic diagnoses in addition to the spell due to SRMD, was only predictive of DP due to somatic diagnoses, not of DP due to mental diagnoses. These differences were also noted for in- and specialised outpatient health care due to mental and somatic diagnoses. In- and specialised outpatient care due to mental diagnoses was only associated with a significantly higher risk of granted DP due to mental diagnoses, but not due to somatic diagnoses. The opposite was true for in- and outpatient care due to somatic diagnoses, which was only predictive of DP due to somatic diagnoses, with one exception. The HR for having had more than the median outpatient care visits due to somatic diagnoses (2001-05) was 1.38, 95\% CI 1.21-1.58 for DP due to mental diagnoses compared to having had no such outpatient care.

The partial likehood ratio test revealed significant interactions between the covariates and age. Analyses have therefore been stratified for age. Multivariate HRs and 95\% CIs related to the various covariates for individuals below and above 45 years of age are shown in Table 4 . Female sex, low education, residing in small towns or 
Table 2 Unadjusted and multivariate adjusted hazard ratios (HR) with $95 \%$ confidence intervals, Cl, for disability pension (DP) in individuals ( $N=36304)$ with sickness absence due to stress related mental diagnoses (SRMD) initiated in $\mathbf{2 0 0 5}$

\begin{tabular}{|c|c|c|c|c|}
\hline Characteristics & Crude HR & $\mathrm{Cl} 95 \%$ & Adjusted HR & $\mathrm{Cl} 95 \%)$ \\
\hline \multicolumn{5}{|l|}{ Socio-demographic characteristics } \\
\hline \multicolumn{5}{|l|}{ Sex } \\
\hline Female & 1.09 & $1.00-1.89$ & 1.15 & $1.05-1.25$ \\
\hline Male & 1 & & 1 & \\
\hline \multicolumn{5}{|l|}{ Age (years) } \\
\hline $17-25$ & 1 & & 1 & \\
\hline $26-35$ & 0.98 & $0.75-1.26$ & 1.09 & $0.84-1.42$ \\
\hline $36-45$ & 1.38 & $1.08-1.76$ & 1.64 & $1.27-2.11$ \\
\hline $46-55$ & 2.29 & $1.79-2.91$ & 2.81 & $2.19-3.60$ \\
\hline $56-65$ & 5.28 & $4.15-6.72$ & 5.30 & $4.15-6.83$ \\
\hline \multicolumn{5}{|l|}{ Education (years) } \\
\hline 0 to 9 & 1.63 & $1.46-1.82$ & 1.33 & $1.18-1.48$ \\
\hline 10 to 12 & 1.02 & $0.94-1.11$ & 1.06 & $0.98-1.16$ \\
\hline Above 12 & 1 & & 1 & \\
\hline \multicolumn{5}{|l|}{ Country of birth* } \\
\hline Sweden & 1 & & 1 & \\
\hline Other Nordic countries & 1.33 & $1.11-1.60$ & 1.16 & $0.96-1.40$ \\
\hline EU25 without Nordic countries & 1.14 & $0.88-1.48$ & 0.87 & $0.66-1.13$ \\
\hline Rest of the world & 1.67 & $1.48-1.89$ & 1.75 & $1.54-1.99$ \\
\hline \multicolumn{5}{|l|}{ Place of residence ${ }^{* *}$} \\
\hline Big cities & 1 & & 1 & \\
\hline Medium sized cities & 1.22 & $1.12-1.34$ & 1.16 & $1.06-1.27$ \\
\hline Small town & 1.35 & $1.23-1.48$ & 1.33 & $1.21-1.47$ \\
\hline \multicolumn{5}{|l|}{ Type of family } \\
\hline Married/cohabiting with children ${ }^{a}$ & 1 & & 1 & \\
\hline Married/cohabiting without children ${ }^{a}$ & 2.60 & $2.34-2.89$ & 1.21 & $1.07-1.36$ \\
\hline Single without children ${ }^{a}$ & 1.45 & $1.32-1.60$ & 1.19 & $1.08-1.32$ \\
\hline Single with children ${ }^{a}$ & 1.17 & $1.04-1.32$ & 1.02 & $0.90-1.15$ \\
\hline \multicolumn{5}{|l|}{ Sickness absence characteristics ${ }^{b}$} \\
\hline \multicolumn{5}{|l|}{ Number of spells } \\
\hline Only mental & 1 & & 1 & \\
\hline 1 spell somatic & 1.65 & $1.47-1.83$ & 1.44 & $1.28-1.61$ \\
\hline$>1$ spell somatic & 1.80 & $1.36-2.24$ & 1.78 & $1.32-2.34$ \\
\hline \multicolumn{5}{|l|}{ Duration (days) $^{c}$} \\
\hline $1-14$ & 1 & & 1 & \\
\hline $15-90$ & 1.10 & $0.96-1.26$ & 1.11 & $0.97-1.28$ \\
\hline $91-180$ & 1.99 & $1.68-2.37$ & 1.99 & $1.66-2.35$ \\
\hline $181-365$ & 6.24 & $5.38-7.23$ & 5.90 & $5.09-6.84$ \\
\hline$>365$ & 20.72 & $18.3-23.5$ & 16.57 & $14.6-18.8$ \\
\hline
\end{tabular}


Table 2 Unadjusted and multivariate adjusted hazard ratios (HR) with $95 \%$ confidence intervals, Cl, for disability pension (DP) in individuals $(N=36$ 304) with sickness absence due to stress related mental diagnoses (SRMD) initiated in 2005 (Continued)

\begin{tabular}{|c|c|c|c|c|}
\hline \multicolumn{5}{|c|}{ Health care characteristics } \\
\hline \multicolumn{5}{|c|}{ Length of hospital stay due to mental diagnoses (days) ${ }^{d}$} \\
\hline No hospital stay & 1 & & 1 & \\
\hline 1 to 8 & 2.29 & $1.88-2.79$ & 1.46 & 1.19-1.79 \\
\hline$>8$ & 3.59 & $3.03-4.26$ & 1.78 & $1.48-2.15$ \\
\hline \multicolumn{5}{|c|}{ Length of hospital stay due to somatic diagnoses (days) ${ }^{d}$} \\
\hline No hospital stay & 1 & & 1 & \\
\hline 1 to 4 & 1.34 & $1.22-1.48$ & 1.06 & $0.96-1.17$ \\
\hline$>4$ & 1.38 & $1.25-1.53$ & 1.08 & $0.97-1.20$ \\
\hline \multicolumn{5}{|c|}{ Outpatient care visit due to mental diagnoses (days) ${ }^{d}$} \\
\hline No visits & 1 & & 1 & \\
\hline 1 & 1.69 & $1.46-1.96$ & 1.29 & $1.12-1.50$ \\
\hline$>1$ & 3.04 & $2.69-3.44$ & 1.52 & $1.32-1.74$ \\
\hline \multicolumn{5}{|c|}{ Outpatient care visit due to somatic diagnoses $(\text { days })^{d}$} \\
\hline No visits & 1 & & 1 & \\
\hline $1-4$ & 1.27 & $1.14-1.42$ & 1.16 & $1.04-1.30$ \\
\hline$>4$ & 2.09 & $1.88-2.33$ & 1.51 & $1.35-1.71$ \\
\hline
\end{tabular}

villages, sick-leave duration exceeding one year and inpatient health care due to mental disorders seemed to be more strongly associated with subsequent granting of disability pension in individuals younger than 45 years than for their older counterparts. On the other hand, living alone or living with a partner but without children and having somatic sick-leave spells were associated with higher HRs for the older cohort.

\section{Discussion}

\section{Main findings}

In this population based cohort study of individuals with a new sickness absence spell due to stress-related mental disorders in 2005, being female, over 35 years of age, with low educational level, having been born outside of Europe (EU25/Nordic countries), living outside big cities or alone were found to be predictors of granted all-cause disability pension during the five subsequent years of follow-up. In addition, long duration of sick-leave spells due to SRMD, additional spells and outpatient care due to somatic diagnoses as well as in- and specialised outpatient care due to mental diagnoses predicted all-cause DP. Female sex, low educational level, living outside big cities, sickness absence spells and inpatient care due to somatic diagnoses turned out to be only predictive of DP due to somatic diagnoses. On the other hand, living alone as well as in- and outpatient care due to mental diagnoses were associated with higher risks for DP due to mental diagnoses.

\section{Methodology}

Among the strengths of this study the large study population consisting of 36546 individuals with a new sickness absence due to SRMD, practically no drop out with a long follow-up of five years and the use of data of good quality should be mentioned [20-22]. The study had further the opportunity to examine a wide variety of sociodemographic factors as well as factors related to sickness absence and health care patterns.

The validity of sickness absence and disability pension diagnoses is often discussed. A previous Swedish study, however, assessed the validity of sickness absence diagnoses and considered it to be good [21]. Moreover, there is an on-going debate regarding the differentiation between stress-related mental disorders and depression [23]. Still, diagnostic guidelines for stress-related mental disorders published by the National Board of Health and Welfare in Sweden determine that in the presence of a depression, depression should be used as the main diagnosis [23]. Moreover, previous research suggests that individuals sickness absent due to stress-related mental disorders differ considerably from the individuals absent 
Table 3 Multivariate adjusted hazard ratios (HR) with $95 \%$ confidence intervals, $\mathrm{Cl}$, for disability pension (DP) due to mental and somatic diagnoses in individuals $(N=36304)$ with sickness absence due to stress related mental diagnoses (SRMD) initiated in 2005

\begin{tabular}{|c|c|c|c|c|}
\hline \multirow[b]{2}{*}{ Characteristics } & \multicolumn{2}{|c|}{ DP mental diagnoses } & \multicolumn{2}{|c|}{ DP somatic diagnoses } \\
\hline & $\begin{array}{l}\text { Adjusted hazard } \\
\text { ratios (HR) }\end{array}$ & $\begin{array}{l}\text { Confidence interval } \\
\text { (CI 95\%) }\end{array}$ & $\begin{array}{l}\text { Adjusted hazard } \\
\text { ratios (HR) }\end{array}$ & $\begin{array}{l}\text { Confidence interval } \\
\text { (Cl 95\%) }\end{array}$ \\
\hline \multicolumn{5}{|l|}{ Socio-demographic characteristics } \\
\hline \multicolumn{5}{|l|}{ Sex } \\
\hline Female & 1.07 & $0.97-1.19$ & 1.36 & $1.14-1.63$ \\
\hline Male & 1 & & 1 & \\
\hline \multicolumn{5}{|l|}{ Age (years) } \\
\hline $17-25$ & 1 & & 1 & \\
\hline $26-35$ & 1.03 & $0.78-1.37$ & 1.32 & $0.67-2.61$ \\
\hline $36-45$ & 1.49 & $1.13-1.97$ & 2.17 & $1.12-4.19$ \\
\hline $46-55$ & 2.20 & $1.67-2.89$ & 6.08 & $3.20-11.53$ \\
\hline $56-65$ & 3.76 & $2.86-4.95$ & 14.59 & $7.70-27.63$ \\
\hline \multicolumn{5}{|l|}{ Education (years) } \\
\hline 0 to 9 & 1.10 & $0.96-1.26$ & 2.14 & $1.73-2.64$ \\
\hline 10 to 12 & 0.94 & $0.86-1.04$ & 1.53 & $1.29-1.82$ \\
\hline Above 12 & 1 & & 1 & \\
\hline \multicolumn{5}{|l|}{ Country of birth* } \\
\hline Sweden & 1 & & 1 & \\
\hline Other Nordic countries & 1.00 & $0.79-1.27$ & 1.51 & $1.12-2.05$ \\
\hline EU25 without Nordic countries & 1.03 & $0.78-1.36$ & 0.39 & $0.17-0.87$ \\
\hline Rest of the world & 1.76 & $1.53-2.03$ & 1.60 & $1.19-2.14$ \\
\hline \multicolumn{5}{|l|}{ Place of residence ${ }^{* *}$} \\
\hline Cities & 1 & & 1 & \\
\hline Medium sized cities & 0.98 & $0.89-1.11$ & 1.84 & $1.53-2.22$ \\
\hline Small towns/villages & 1.18 & $1.06-1.32$ & 1.95 & $1.60-2.38$ \\
\hline \multicolumn{5}{|l|}{ Type of family } \\
\hline Married/cohabiting with children ${ }^{a}$ & 1 & & 1 & \\
\hline Married/cohabiting without children ${ }^{a}$ & 1.22 & $1.06-1.39$ & 1.14 & $0.91-1.42$ \\
\hline Single people without children ${ }^{a}$ & 1.17 & $1.04-1.31$ & 1.19 & $0.97-1.46$ \\
\hline Single people with children ${ }^{a}$ & 1.04 & $0.91-1.19$ & 0.98 & $0.77-1.26$ \\
\hline \multicolumn{5}{|l|}{ Sickness absence characteristics ${ }^{b}$} \\
\hline \multicolumn{5}{|l|}{ Number of spells } \\
\hline Only mental & 1 & & 1 & \\
\hline 1 spell somatic & 1.11 & $0.96-1.28$ & 2.31 & $1.93-2.75$ \\
\hline$>1$ spell somatic & 1.25 & $0.84-1.88$ & 2.97 & $1.97-4.49$ \\
\hline \multicolumn{5}{|l|}{ Duration (days) ${ }^{c}$} \\
\hline $1-14$ & 1 & & 1 & \\
\hline $15-90$ & 1.19 & $0.99-1.43$ & 1.03 & $0.84-1.27$ \\
\hline $91-180$ & 2.76 & $2.23-3.42$ & 1.04 & $0.75-1.44$ \\
\hline $181-365$ & 7.89 & $6.54-9.53$ & 3.53 & $2.74-4.54$ \\
\hline$>365$ & 24.99 & $21.17-29.51$ & 6.50 & $5.22-8.11$ \\
\hline
\end{tabular}


Table 3 Multivariate adjusted hazard ratios (HR) with $95 \%$ confidence intervals, $\mathrm{Cl}$, for disability pension (DP) due to mental and somatic diagnoses in individuals $(\mathrm{N}=36304)$ with sickness absence due to stress related mental diagnoses (SRMD) initiated in 2005 (Continued)

\begin{tabular}{|c|c|c|c|c|}
\hline \multicolumn{5}{|c|}{ Health care characteristics } \\
\hline \multicolumn{5}{|c|}{ Length of hospital stay due to mental diagnoses (days) ${ }^{d}$} \\
\hline No hospital stay & 1 & & 1 & \\
\hline 1 to 8 & 1.68 & $1.35-2.10$ & 0.66 & $0.36-1.20$ \\
\hline$>8$ & 2.03 & $1.67-2.47$ & 0.71 & $0.37-1.36$ \\
\hline \multicolumn{5}{|c|}{ Length of hospital stay due to somatic diagnoses (days) ${ }^{d}$} \\
\hline No hospital stay & 1 & & 1 & \\
\hline 1 to 4 & 0.99 & $0.87-1.12$ & 1.31 & $1.08-1.59$ \\
\hline$>4$ & 0.92 & $0.80-1.05$ & 1.58 & $1.30-1.93$ \\
\hline \multicolumn{5}{|c|}{ Outpatient care visits due to mental diagnoses (days) ${ }^{d}$} \\
\hline No visits & 1 & & 1 & \\
\hline 1 & 1.41 & $1.19-1.66$ & 1.05 & $0.75-1.46$ \\
\hline$>1$ & 1.68 & $1.44-1.95$ & 0.96 & $0.65-1.39$ \\
\hline \multicolumn{5}{|c|}{ Outpatient care visits due to somatic diagnoses (days) ${ }^{d}$} \\
\hline No visits & 1 & & 1 & \\
\hline $1-4$ & 1.11 & $0.98-1.26$ & 1.33 & $1.06-1.67$ \\
\hline$>4$ & 1.38 & $1.21-1.58$ & 1.98 & $1.56-2.51$ \\
\hline
\end{tabular}

" "Other Nordic countries"; Rest of the world includes all remaining countries. **Place of residence: big cities: Stockholm, Gothenburg and Malmö; medium-sized cities: cities with more than 90000 inhabitants within $30 \mathrm{~km}$ distance from the centre of the city; ${ }^{\mathrm{a} C h i l d r e n ~ l i v i n g}$ at home; ${ }^{\mathrm{b}}$ Sickness absence due to SRMD (stress related mental diagnoses); ' Length of the first sickness absence spell due to stress related mental diagnoses; ${ }^{\mathrm{d}}$ Cut-offs are based on the median, inpatient care from 2000-2005; outpatient care from 2001-2005. Adjusted HRs indicate that all variables have been mutually adjusted for each other.

due to depression with regard to their risk of disability pension and the degree of previous health-care consumption [24].

We may have missed individuals with a stress-related mental disorder as a secondary diagnosis, as in the Social Insurance Agency's register only the main diagnosis was recorded for any sickness absence spell. Also any comorbid disorders not covered by the information in the in- and specialised outpatient care register may have been missed. We therefore anticipate that only mental and somatic disorders of greater medical severity were covered. This study investigated a wide range of different risk factors for disability pension in individuals sickness absent due to stress-related mental disorders. Still, additional risk factors, like e.g. overweight, might have an association with subsequent labor market exit and may have an effect on the estimates of the studied covariates [25]. However, such information was not available in the database used.

We found that female sex, increasing age, low education and living in small towns/villages were associated with an increased risk of disability pension. These findings are consistent with the findings of previous studies of individuals on long term sickness absence due to mental disorders and individuals with outpatient care due to depression $[6,26]$. Analyses of diagnosis-specific
DP revealed that low education was not associated with an increased risk for DP due to mental diagnoses. These findings are comparable to results from a previous study [26]. On the other hand, low education was clearly predictive of DP due to somatic disorders. Further studies are warranted to scrutinise these relations with regard to additional determinants of socio-economic status and specific somatic diagnoses of DP.

Being born in a country outside Northern Europe and outside EU25 was predictive for DP, both due to mental and somatic diagnoses. The finding that first generation immigrants have a higher risk for disability pension was earlier reported in studies based on the general population [27] and individuals on long term sick-leave [12], but to our best knowledge not on individuals sickness absent due to SRMD. Previously, differences in health care access and utilisation as well as working conditions and income in first generation immigrants compared to the native population have been reported [28]. In our analyses, we controlled for differences in specialised health care, socio-economic status and a range of additional socio-demographic factors. Future studies are warranted in order to elucidate mechanisms behind the higher risk of disability pension among immigrants with sickness absence due to SRMD, particularly those with a birth country outside Europe (EU25/Nordic countries). 
Table 4 Multivariate adjusted hazard ratios (HR) with $95 \%$ confidence intervals, Cl, for disability pension (DP) in individuals ( $N=36304$ ) with sickness absence due to stress-related mental diagnoses (SRMD) initiated in 2005, stratified for age $(<=$ and $>45$ years $)$

\begin{tabular}{|c|c|c|c|c|}
\hline \multirow[b]{2}{*}{ Characteristics } & \multicolumn{2}{|c|}{$<=45$ years } & \multicolumn{2}{|c|}{$>45$ years } \\
\hline & $\begin{array}{l}\text { Adjusted hazard } \\
\text { ratios (HR) }\end{array}$ & $\begin{array}{l}\text { Confidence interval } \\
\text { (Cl 95\%) }\end{array}$ & $\begin{array}{l}\text { Adjusted hazard } \\
\text { ratios (HR) }\end{array}$ & $\begin{array}{l}\text { Confidence interval } \\
\text { (CI 95\%) }\end{array}$ \\
\hline \multicolumn{5}{|l|}{ Socio-demographic characteristics } \\
\hline \multicolumn{5}{|l|}{ Sex } \\
\hline Female & 1.26 & $1.07-1.47$ & 1.03 & $0.92-1.15$ \\
\hline Male & 1 & & 1 & \\
\hline \multicolumn{5}{|l|}{ Education (years) } \\
\hline 0 to 9 & 1.47 & $1.21-1.78$ & 1.22 & $1.06-1.40$ \\
\hline 10 to 12 & 1.06 & $0.92-1.23$ & 0.99 & $0.90-1.11$ \\
\hline Above 12 & 1 & & 1 & \\
\hline \multicolumn{5}{|l|}{ Country of birth* } \\
\hline Sweden & 1 & & 1 & \\
\hline Other Nordic countries & 1.36 & $0.95-1.56$ & 1.11 & $0.89-1.37$ \\
\hline EU25 without Nordic countries & 1.04 & $0.63-1.71$ & 0.83 & $0.61-1.14$ \\
\hline Rest of the world & 1.59 & $1.34-1.89$ & 1.78 & $1.48-2.14$ \\
\hline \multicolumn{5}{|l|}{ Place of residence ${ }^{* *}$} \\
\hline Cities & 1 & & 1 & \\
\hline Medium sized cities & 1.18 & $1.01-1.37$ & 1.14 & $1.01-1.27$ \\
\hline Small towns/villages & 1.56 & $1.33-1.84$ & 1.19 & $1.05-1.35$ \\
\hline \multicolumn{5}{|l|}{ Type of family } \\
\hline Married/cohabiting with children ${ }^{a}$ & 1 & & 1 & \\
\hline Married/cohabiting without children ${ }^{a}$ & 0.82 & $0.57-1.18$ & 1.73 & $1.52-1.97$ \\
\hline Single people without children ${ }^{\mathrm{a}}$ & 1.06 & $0.91-1.23$ & 1.49 & $1.31-1.71$ \\
\hline Single people with children ${ }^{a}$ & 1.02 & $0.86-1.20$ & 1.01 & $0.84-1.19$ \\
\hline \multicolumn{5}{|l|}{ Sickness absence characteristics ${ }^{b}$} \\
\hline \multicolumn{5}{|l|}{ Number of spells } \\
\hline Only mental & 1 & & 1 & \\
\hline 1 spell somatic & 1.29 & $1.07-1.56$ & 1.57 & $1.37-1.81$ \\
\hline$>1$ spell somatic & 1.09 & $0.63-1.90$ & 2.33 & $1.66-3.26$ \\
\hline \multicolumn{5}{|l|}{ Duration (days) ${ }^{c}$} \\
\hline $1-14$ & 1 & & 1 & \\
\hline $15-90$ & 1.24 & $0.97-1.59$ & 1.08 & $0.91-1.28$ \\
\hline $91-180$ & 1.81 & $1.32-2.48$ & 2.12 & $1.73-2.61$ \\
\hline $181-365$ & 5.33 & $4.06-7.01$ & 6.44 & $5.40-7.67$ \\
\hline$>365$ & 23.04 & $18.35-28.91$ & 15.04 & $12.89-17.56$ \\
\hline \multicolumn{5}{|l|}{ Health care characteristics } \\
\hline \multicolumn{5}{|c|}{ Length of hospital stay due to mental diagnoses (days) ${ }^{d}$} \\
\hline No hospital stay & 1 & & 1 & \\
\hline 1 to 8 & 1.83 & $1.41-2.39$ & 1.01 & $0.72-1.43$ \\
\hline$>8$ & 2.10 & $1.70-2.78$ & 1.43 & $1.08-1.90$ \\
\hline
\end{tabular}




\section{Table 4 Multivariate adjusted hazard ratios (HR) with $95 \%$ confidence intervals, Cl, for disability pension (DP) in individuals ( $\mathrm{N}=36304$ ) with sickness absence due to stress-related mental diagnoses (SRMD) initiated in 2005, stratified for age (<= and $>\mathbf{4 5}$ years) (Continued)}

\begin{tabular}{|c|c|c|c|c|}
\hline \multicolumn{5}{|c|}{ Length of hospital stay due to somatic diagnoses (days) $^{d}$} \\
\hline No hospital stay & 1 & & 1 & \\
\hline 1 to 4 & 1.09 & $0.92-1.28$ & 1.02 & $0.89-1.16$ \\
\hline$>4$ & 1.08 & $0.91-1.28$ & 1.03 & $0.89-1.19$ \\
\hline \multicolumn{5}{|c|}{ Outpatient care visits due to mental diagnoses (days) ${ }^{d}$} \\
\hline No visits & 1 & & 1 & \\
\hline 1 & 1.17 & $0.94-1.47$ & 1.33 & $1.09-1.62$ \\
\hline$>1$ & 1.51 & $1.25-1.83$ & 1.30 & $1.05-1.60$ \\
\hline \multicolumn{5}{|c|}{ Outpatient care visits due to somatic diagnoses (days) $^{d}$} \\
\hline No visits & 1 & & 1 & \\
\hline $1-4$ & 1.07 & $0.88-1.30$ & 1.23 & $1.08-1.41$ \\
\hline$>4$ & 1.52 & $1.24-1.86$ & 1.53 & $1.32-1.77$ \\
\hline
\end{tabular}

" "Other Nordic countries include Denmark, Norway and Finland; EU25 includes all countries included in the European Union in 2006 without Sweden and without "Other Nordic countries"; Rest of the world includes all remaining countries. **Place of residence: big cities: Stockholm, Gothenburg and Malmö; medium-sized cities: cities with more than 90000 inhabitants within $30 \mathrm{~km}$ distance from the centre of the city; ${ }^{\mathrm{a} C h i l d r e n ~ l i v i n g ~ a t ~ h o m e ; ~}{ }^{\mathrm{b}}$ Sickness absence due to SRMD (stress-related mental diagnoses); ' Length of the first sickness absence spell due to stress-related mental diagnoses; ${ }^{\mathrm{d}} \mathrm{Cut}$-offs are based on the median, inpatient care from 2000-2005; outpatient care from 2001-2005. Adjusted HRs indicate that all variables have been mutually adjusted for each other.

The risk of being granted disability pension was found to increase with increasing duration of sick leave and number of mental sick-leave spells, which is consistent with previous reports based on the general population and occupational cohorts [10,11,29-31]. Duration of sickness absence and several spells may not solely be a measure of the chronicity and severity of the underlying disorder [32], but might also be a marker of inadequate treatment and rehabilitation efforts [32]. Moreover, increasing duration of sickness absence might be associated with social isolation and increased alcohol consumption [33], which in turn may increase the risk of disability pension. In contrast to the association of sickness absences exceeding three months with disability pension, sickness absence shorter than three months was not associated with an increased risk of DP. Future studies are needed to investigate the optimal, individually varying duration of sickness absence due to stress-related mental disorders in order to prevent early exit from the labour market.

Frequent and long duration of specialised health care due to mental diagnoses predicted all-cause DP and DP due to mental disorders, but not DP due to somatic disorders. These findings are consistent with previous studies, indicating that frequent health care consumption was related with an increased risk of DP [34]. Comorbid somatic disorders, measured as additional sickness absence spells and health care due to somatic diagnoses, were risk factors for all-cause DP, particularly for DP due to somatic disorders. Further research is required to evaluate possible implication of these findings for treatment and rehabilitation after sick-leave due to stressrelated mental disorders.
We found that the effect of risk markers varied with age. To the best of our knowledge, this has not been reported in studies on sickness absentees due to SRMD before. In younger individuals, risk markers related to the severity of the underlying disease (including mental inpatient care and long sickness absences) were more strongly associated with an increased risk of granted disability pension than among older individuals. Also if young individuals lived outside big cities and had a low educational level, they had a higher risk of being granted a disability pension during the five years of follow-up than their older counterparts. The associations in our study remained after adjustment of differences in specialized health care and a number of other socio-demographic factors. Socio-economic and regional differences in health care, work-place rehabilitation and labor market policies may be related to these findings and should be elucidated in future studies.

\section{Conclusions}

Female sex, low education, increasing age, born outside Europe (EU25/Nordic countries), living in rural areas or alone, long duration of sickness absence, comorbidity with somatic disorders and severity of the underlying mental disorder increased the risk of granted DP in individuals with sick leave due to stress-related mental disorders. Some different patterns emerged for risk factors related to granted disability pension due to mental and somatic diagnoses and for younger and older individuals. The variation in the effect of risk markers with regard to age and diagnosis of disability pension speaks in favour of the importance of a person-centered approach in treatment and rehabilitation. 


\section{Competing interests}

The authors declare that they have no competing interest.

\section{Authors' contributions}

EMR is responsible for the core idea and study design. KIA and EMR carried out the data analyses and drafted the manuscripts. EMR, KIA and AP contributed to successive drafts and agreed on the final version of the manuscript. All authors read and approved the final manuscript.

\section{Acknowledgements}

We thank Linnea Kjeldgård, statistician, for data management. This study was funded by the Swedish Research Council.

\section{Author details}

'Division of Insurance Medicine, Department of Clinical Neuroscience, Karolinska Institutet, Berzelius väg 3, SE- 17177 Stockholm, Sweden. ${ }^{2}$ Department of Public Health Sciences, Karolinska Institutet, Stockholm, Sweden. ${ }^{3}$ Stockholm University, Stockholm Stress Centre, Stockholm, Sweden.

Received: 24 January 2014 Accepted: 24 July 2014

Published: 7 August 2014

\section{References}

1. Järvisalo J, Kansaneläkelaitos (Finland): Mental disorders as a major challenge in prevention of work disability: experiences in Finland, Germany, the Netherlands and Sweden. Helsinki: KELA; 2005.

2. Försäkringskassan (Swedish Social Insurance Agency): Sjukfrånvaro i psykiska diagnoser: Delrapport: (sickness absence due to mental diagnoses: first report). 2013.

3. WHO: The ICD classification of mental and behavioural disorders: clinical descriptions and diagnostic guidelines. World Health Organization; 1997.

4. Hultin $\mathrm{H}$, Lindholm C, Moller J: Is there an association between long-term sick leave and disability pension and unemployment beyond the effect of health status?-a cohort study. PloS One 2012, 7(4):e35614. Research Support, Non-U.S. Gov't.

5. Alexanderson K, Kivimaki M, Ferrie JE, Westerlund H, Vahtera J, Singh-Manoux A, Melchior M, Zins M, Goldberg M, Head J: Diagnosis-specific sick leave as a long-term predictor of disability pension: a 13-year follow-up of the GAZEL cohort study. J Epidemiol Commun H 2012, 66(2):155-9.

6. Bratberg E, Gjesdal S, Maeland JG: Sickness absence with psychiatric diagnoses: individual and contextual predictors of permanent disability. Health Place 2009, 15(1):308-14.

7. Gjesdal S, Ringdal PR, Haug K, Maeland JG: Long-term sickness absence and disability pension with psychiatric diagnoses: a population-based cohort study. Nord J Psychiatry 2008, 62(4):294-301.

8. Gjesdal S, Haug K, Ringdal P, Maeland JG, Hagberg J, Roraas T, Vollset SE, Alexanderson K: Sickness absence with musculoskeletal or mental diagnoses, transition into disability pension and all-cause mortality: a 9-year prospective cohort study. Scand J Public Health 2009, 37(4):387-94.

9. Kivimaki M, Ferrie JE, Hagberg J, Head J, Westerlund H, Vahtera J, Alexanderson K: Diagnosis-specific sick leave as a risk marker for disability pension in a Swedish population. J Epidemiol Community Health 2007, 61(10):915-20.

10. Gjesdal S, Ringdal PR, Haug K, Maeland JG: Predictors of disability pension in long-term sickness absence: results from a population-based and prospective study in Norway 1994-1999. Eur J Pub Health 2004, 14(4):398-405.

11. Gjesdal S, Bratberg E: Diagnosis and duration of sickness absence as predictors for disability pension: results from a three-year, multi-register based* $^{*}$ and prospective study. Scand J Public Health 2003, 31(4):246-54

12. Karlsson NE, Carstensen JM, Gjesdal S, Alexanderson KA: Risk factors for disability pension in a population-based cohort of men and women on long-term sick leave in Sweden. Eur J Pub Health 2008, 18(3):224-31.

13. Mittendorfer-Rutz E, Kjeldgård L, Wikman A, Alexanderson K: Sjukskrivning i psykiska diagnoser och risk för att få sjuk-eller aktivitetsersättning eller för förtida död (Sick leave in psychiatric diagnoses and the risk of disability pension/sick leave and pre-mature death) (In Swedish). Stockholm, Sweden: Department of Clinical Neuroscience, Karolinska Institutet; 2011.

14. OECD: Transforming disability into ability: policies to promote work and income security for disabled people, Paris. 2003.
15. Pietilainen O, Laaksonen M, Rahkonen O, Lahelma E: Self-rated health as a predictor of disability retirement - the contribution of III-health and working conditions. PLOS One 2011, 20:6(9)

16. Karpansalo M, Manninen P, Kauhanen J, Lakka TA, Salonen JT: Perceived health as a predictor of early retirement. Scand J Work Environ Health 2004, 30(4):287-92.

17. OECD: Mental health and work: Sweden. In OECD publishing. Organisation for Economic Co-operation and Development; 2013.

18. Försäkringskassan (Swedish Social Insurance Agency): Social insurance in figures, 2012 (The social insurance agency). 2012.

19. Försäkringskassan (Swedish Social Insurance Agency): Sjukpenning, 2012 (Sickness benefit, 2012) (in Swedish). 2012

20. Ludvigsson JF, Andersson E, Ekbom A, Feychting M, Kim JL, Reuterwall C, Heurgren M, Olausson PO: External review and validation of the Swedish national inpatient register. BMC Public Health 2011, 11:450.

21. Ljungdahl LO, Bjurulf P: The accordance of diagnoses in a computerized sick-leave register with doctor's certificates and medical records. Scand J Soc Med 1991, 19(3):148-53.

22. Socialstyrelsen: 2009. The Cause of Death Register Available from: www.social-styrelsen.se.

23. The National Board of Health and Welfare (Socialstyrelsen): Fatigue syndrome, stress related psychiatric disorders (Utmattningssyndrom stressrelaterad psykisk ohälsa). Stockholm: The National Board of Health and Welfare (Socialstyrelsen); 2003.

24. Mittendorfer Rutz E, Kjeldgård L, Runeson B, Perski3 A, Melchior M, Head J, Alexanderson K: Sickness absence due to specific mental diagnoses and all-cause and cause-specific mortality: a cohort study of 4.9 million inhabitants of Sweden. PLoS One 2012, 7(9).

25. Claessen $\mathrm{H}$, Arndt V, Drath C, Brenner H: Overweight, obesity and risk of work disability: a cohort study of construction workers in Germany. Occup Environ Med 2009, 66(6):402-9. doi:10.1136/oem.2008.042440. Epub 2009 Feb 5.

26. Samuelsson A, Alexanderson K, Ropponen A, Lichtenstein P, Svedberg P: Incidence of disability pension and associations with socio-demographic factors in a Swedish twin cohort. Soc Psychiatry Psychiatr Epidemiol 2012, 47(12):1999-2009.

27. Johansson B, Helgesson M, Lundberg I, Nordquist T, Leijon O, Lindberg P, Vingard E: Work and health among immigrants and native Swedes 1990-2008: a register-based study on hospitalization for common potentially work-related disorders, disability pension and mortality. BMC Public Health 2012, 12:845.

28. Meershoek A, Krumeich A, Vos R: The construction of ethnic differences in work incapacity risks: analysing ordering practices of physicians in the Netherlands. Soc Sci Med 2011, 72(1):15-22.

29. Selander J, Marnetoft SU: Risk factors for disability pension among unemployed women on long-term sick-leave. Int J Rehabil Res 1999, 22(4):277-82.

30. Borg K, Hensing G, Alexanderson K: Predictive factors for disability pension - an 11-year follow up of young persons on sick leave due to neck, shoulder, or back diagnoses. Scand J Public Healt 2001, 29(2):104-12.

31. Gjesdal S, Bratberg E: The role of gender in long-term sickness absence and transition to permanent disability benefits - results from a multiregister based, prospective study in Norway 1990-1995. Eur J Pub Health 2002, 12(3):180-6.

32. Bultmann U, Rugulies R, Lund T, Christensen KB, Labriola M, Burr H: Depressive symptoms and the risk of long-term sickness absence: a prospective study among 4747 employees in Denmark. Soc Psychiatry Psychiatr Epidemiol 2006, 41(11):875-80.

33. Vingard E, Alexanderson $K$, Norlund A: Chapter 9: consequences of being on sick leave. Scand J Public Health Suppl [Review] 2004, 63:207-15.

34. Wallman T, Burell G, Kullman S, Svardsudd K: Health care utilisation before and after retirement due to illness - A 13-year population-based followup study of prematurely retired men and referents from the general population. Scand J Prim Health 2004, 22(2):95-100.

\section{doi:10.1186/1471-2458-14-805}

Cite this article as: Ishtiak-Ahmed et al:: Risk markers of all-cause and diagnosis-specific disability pension - a prospective cohort study of individuals sickness absent due to stress-related mental disorders. BMC Public Health 2014 14:805. 